Universidade Tecnológica Federal do Paraná - UTFPR

Campus Ponta Grossa - Paraná - Brasil

ISSN: $1981-3686 /$ v. 05, n. $02:$ p. $559-572,2011$

D.O.I.: $10.3895 / \mathrm{S} 1981-36862011000200008$
Revista Brasileira de Tecnologia

Agroindustrial

\title{
ELABORAÇÃO E CARACTERIZAÇÃO SENSORIAL E FUNCIONAL DE UM LICOR TÍPICO AMAZÔNICO A BASE DE AÇAÍ (Euterpe oleracea)
}

\author{
PREPARATION AND CHARACTERIZATION SENSORY AND \\ FUNCTIONAL PROPERTIES OF A TYPICAL LIQUOR AMAZONIAN \\ ACAI-BASED (Euterpe oleracea)
}

\author{
Yamila Fernandes Mota Alves ${ }^{1}$; Xaene Maria Fernandes Duarte Mendonça ${ }^{2}$ \\ ${ }^{1,2}$ Universidade Federal do Pará - UFPA - Belém - Brasil yamilalves@ yahoo.com.br
}

\begin{abstract}
Resumo
O açaí é considerado um fruto com importante composição nutricional destacando-se pelo seu teor significativo de antocianinas, um flavonóide que apresenta efeitos benéficos em relação à saúde humana, já que possui propriedades antioxidantes comprovadas cientificamente. Objetivou-se neste estudo elaborar e caracterizar sensorial e funcionalmente um licor a base de açaí com diferentes concentrações de açaí e aguardente. As formulações foram submetidas a testes sensoriais de caracterização, preferência e aceitabilidade e os dados obtidos foram analisados por ANOVA $e$ TUKEY ao nível de 5\% usando o software BIOESTAT versão 5.0 (AYRES, 2007). Para a análise da caracterização funcional, foi analisado o teor de antocianinas em laboratório credenciado. Em relação aos resultados, no teste de caracterização as formulações selecionadas foram: F5 (50:50\%); F6 (60:40\%) e F7 (70:30\%) (açaí: aguardente). Em seguida, no teste de preferência, as formulações F6 e F7 somente diferenciaram-se nos atributos consistência e impressão global onde a amostra $F 7$ obteve as maiores médias (7,12 e 7,24). Esta então foi a escolhida e submetida ao teste de aceitabilidade a qual obteve $98 \%$ de aceitação entre os provadores e 78,6\% afirmaram que comprariam o produto. Na análise da caracterização funcional, o licor F7 e F6 obtiveram teores de antocianinas superiores aos valores médios encontrados em sucos de uvas e em vinhos tintos. Desta forma, os resultados da análise sensorial demonstraram que o produto elaborado possui grande potencial de consumo no mercado e também pode ser classificado como uma bebida com boa fonte de compostos fenólicos.
\end{abstract}

Palavras-chave: antocianinas; avaliação sensorial; antioxidantes.

\section{Introdução}

$\mathrm{O}$ açaizeiro é uma palmeira que fornece dois produtos alimentares essenciais: o palmito e os frutos, a partir dos quais a bebida açaí é elaborada. Essa bebida destina-se principalmente a um mercado local e totalmente informal e constitui a base da renda de dezenas de milhares de famílias. 
Desde o início dos anos 1990, o açaí fez sua entrada maciça e brutal sobre o mercado externo, prefigurando o desenvolvimento de uma nova produção de renda (ROGEZ, 2000).

O açaí (Euterpe oleracea) é um dos produtos mais ricos em antocianinas além de representar uma importante fonte de lipídios, proteínas, fibras, minerais $(\mathrm{Mn}, \mathrm{Cu}, \mathrm{Cr}, \mathrm{B})$ e vitaminas. O alto teor de lipídio do açaí confere ao produto um elevado valor energético (SANTOS et al., 2008).

As antocianinas (das palavras gregas "anthos", flor e "kianos", azul) são pigmentos solúveis em água, intensamente coloridos e amplamente distribuídos na natureza, sendo responsável pela maioria das cores azul, violeta e todas as tonalidades de vermelho que aparecem em flores, frutos e algumas folhas, caules e raízes de plantas. Esse pigmento, bem como os flavonóis, as flavonas, as catequinas e as flavanonas são grupos importantes dos flavonóides (BASHO, 2010).

Já no estudo de Sampaio (2006), afirma-se ainda que os flavonóides possuam uma série de propriedades farmacológicas que os fazem atuarem sobre sistemas biológicos. Conseqüentemente, muitas dessas propriedades atuam de forma benéfica para a saúde humana.

As antocianinas possuem propriedades farmacológicas e propriedades medicinais, incluindo anticarcinogênica, antiinflamatória e antimicrobiana, prevenindo a oxidação de proteínas de baixa densidade (LDL), enfermidades cardiovasculares e doenças neurológicas (MENEZES et al., 2008).

No estudo de Gris et al (2004), observou-se que tais pigmentos não vem sendo utilizados nas indústrias de alimentos por serem susceptíveis a mudanças ou perdas de coloração frente a fatores físico-químicos, sendo indicados para alimentos não submetidos à elevadas temperaturas, durante o processamento, com tempo curto de armazenamento e embalados de forma que a exposição à luz, ao oxigênio e à umidade sejam reduzidas.

Nas frutas e legumes, as antocianinas são geralmente localizadas nas camadas celulares externas (no epicarpo e no parênquima). Aproveita-se desta propriedade para sua extração. Com efeito, os solventes utilizados devem poder desnaturar as paredes e membranas das células e dissolver os pigmentos. Numerosos tipos de solvente são mencionados na literatura. O metanol é o mais usado para fins analíticos; o etanol, o ácido acético $(0,01 \mathrm{M})$, o $\mathrm{SO}_{2}$ aquoso ou uma combinação destes são os mais comuns à escala industrial (ROGEZ, 2000).

Geocze (2007) afirma que uma forma refinada de utilizar o fruto, a fim de extrair e conservar o seu teor fenólico é a elaboração de um licor que se dá com a imersão deste em aguardente por um período mínimo de sete dias. Já no estudo de Asquieri et al. (2008) o autor assegura que existe também a necessidade de se desenvolver novos fermentados para diminuir a perda de frutas e aumentar a renda do agricultor. 
Licor é uma palavra de origem latina "lique facere" que significa fundido ou dissolvido em líquido. Normalmente, produzidos por maceração ou por uma mistura conjunta de diferentes componentes. O número e estilos deste produto são enormes, variando de produtos muito fortes, "tradicionais", ou produtos mais leves, "especiais", tais como os "brands", licores cremosos e aperitivos. O licor tradicional contém cerca de $35 \%$ a $45 \%$ de álcool por volume, entretanto, muito dos novos licores possuem um teor alcoólico mais baixo, em torno de 20\% (v/v) (GEOCZE, 2007).

Segundo a lei n8.918 de 14 de julho de 1994, que dispõe sobre a padronização, a classificação, o registro, a inspeção, a produção e a fiscalização de bebidas, licor é a bebida com graduação alcoólica de quinze a cinqüenta e quatro por cento em volume, a vinte graus Celsius $\left({ }^{\circ} \mathrm{C}\right)$, com percentual de açúcar superior a trinta gramas por litro.

O avanço dos conhecimentos sobre a relação entre alimentação e saúde, bem como os elevados custos da saúde pública e a busca permanente da indústria por inovações têm gerado novos produtos, cujas funções pretendem ir além do conhecido papel nutricional dos alimentos (NETZLAFF et al., 2009).

Diante do exposto, entende-se que o estudo de métodos de elaboração de licores com alta concentração de compostos fenólicos torna-se relevante. O objetivo deste estudo foi elaborar e caracterizar sensorial e funcionalmente um licor típico amazônico a base de açaí.

\section{Material e Métodos}

O licor, denominado de "licor típico amazônico a base de açaí" foi elaborado no Laboratório de Técnica Dietética (LTD) da Faculdade de Nutrição - FANUT/ICS/UFPA. As formulações foram submetidas a testes sensoriais de caracterização, preferência e de aceitabilidade, estes dois últimos por meio de testes de escala hedônica de nove e sete pontos, respectivamente.

As tabelas e a análise estatística foram realizadas através dos programas Microsoft Excel e BioEstat 5.0 (AYRES, 2007), concomitantemente. Foi utilizada a análise de variância (ANOVA) para comparar a magnitude das variações entre as formulações do "licor típico amazônico a base de açaí" analisadas, sendo este complementado com o teste Tukey para verificar a diferença entre as médias dos atributos, utilizando-se um nível de decisão alfa de 0,05 .

Esses testes foram realizados mediante aprovação do Comitê de Ética em Pesquisa envolvendo seres humanos, da Universidade Estadual do Pará - Cep/Campus IV/CCBS cujo no do protocolo da pesquisa foi 0033.0.321.000-10. O estudo recebeu autorização da direção da FANUT/ICS/UFPA e dos discentes, docentes e funcionários que participaram dos respectivos testes, mediante assinatura do TCLE. 
Para o cálculo da amostra foram utilizados os números totais de 265 alunos matriculados, 28 professores efetivos e 07 funcionários do ICS. A amostra foi calculada com base na fórmula: $\mathrm{n}=$ $\left(S^{2}\right.$.P.Q.N) / $E^{2}$.(N-1) + S.P.Q, proposta pelo Instituto do Desenvolvimento Econômico Social do Pará (IDESP, 1993), na qual serão considerados um nível de confiança (S) igual a 1, a porcentagem de ocorrência do fenômeno (P) de 50\%, a porcentagem complementar (Q) equivalente a 100-P, um erro máximo permitido (E) igual a 5\% e o tamanho da população (N) igual a 300. Como resultado obteve-se uma amostra (n) de 103 provadores.

A análise de caracterização funcional foi realizada em laboratório credenciado e os resultados foram expressos por meio de um laudo, emitido pelo técnico responsável.

\section{Elaboração das formulações}

Foram elaboradas oito formulações do "licor típico amazônico a base de açaí", após uma sequiência de ensaios preliminares, que diferenciaram entre si pela composição dos ingredientes: açaí e aguardente, conforme mostra a Tabela 1.

Tabela 1 - Formulações e respectivas concentrações de açaí e aguardente nas formulações do licor.

\begin{tabular}{lrcccccccc}
\hline & \multicolumn{3}{c}{ Ingredientes } & \multicolumn{7}{c}{ Formulações* } \\
& F0 & F1 & F2 & F3 & F4 & F5 & F6 & F7 & F8 \\
\hline Aguardente & 100,0 & 90,0 & 80,0 & 70,0 & 60,0 & 50,0 & 40,0 & 30,0 & 20,0 \\
Açaí & 0,0 & 10,0 & 20,0 & 30,0 & 40,0 & 50,0 & 60,0 & 70,0 & 80,0 \\
Açúcar & 30,0 & 30,0 & 30,0 & 30,0 & 30,0 & 30,0 & 30,0 & 30,0 & 30,0 \\
\hline
\end{tabular}

*Porcentagem dos ingredientes em relação a $100 \%$ do volume total do licor típico amazônico a base de açaí.

O fruto in natura foi adquirido no mercado municipal de Belém "Ver o Peso" para a elaboração das formulações. A aguardente da marca Pirassununga 51 e o açúcar de cana refinado especial da marca União, foram adquiridos em supermercado local.

Para a elaboração das formulações do licor, o açaí foi esmagado manualmente com o rompimento da casca, mas sem injúria da semente, na quantidade indicada pela formulação. Após este esmagamento, o açaí ficou em maceração alcoólica na quantidade de aguardente calculada para cada amostra em um recipiente de vidro esterilizado e coberto por papel alumínio por um período mínimo de 07 dias.

Depois desta infusão realizada, o licor passou por um processo de filtração para retenção de partículas indesejáveis. Em seguida, foi processado o xarope que é o aquecimento do açúcar em água, no forno de temperatura média por aproximadamente 8 minutos. O xarope foi então misturado 
à infusão em temperatura ambiente e assim, armazenado em vasilhame de vidro escuro. A maturação foi de 15 dias para o licor adquirir o sabor, consistência e odor esperado. As etapas do preparo das formulações do licor a base de açaí estão descritas no diagrama, conforme demonstrado na Figura 1.

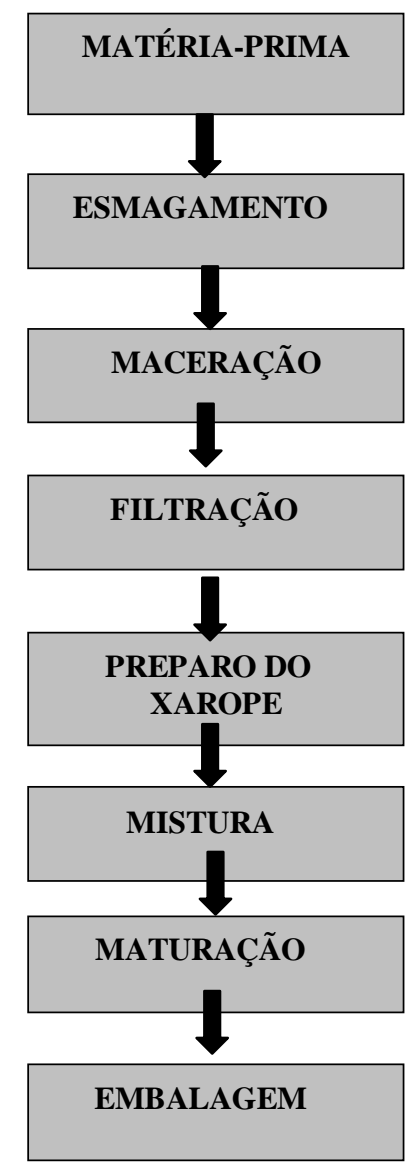

(180 ${ }^{\circ} \mathrm{C}$ por 08 min. e/ou 2 min.)

Figura 1: Diagrama da elaboração das formulações do licor típico amazônico a base de açaí.

\section{Caracterização Sensorial}

A caracterização sensorial foi realizada por meio da aplicação de três testes sendo eles: caracterização, preferência e aceitabilidade. O teste de caracterização do "licor típico amazônico a base de açaí" foi realizado com 05 provadores treinados, de ambos os senxos. As formulações foram servidas em copos descartáveis de $50 \mathrm{~mL}$, codificados com número aleatórios de três digitos, em quantidade aproximada de 10mL acompanhados de água mineral à temperatura ambiente para a limpeza do palato e pó de café para neutralização dos odores. Os atributos avaliados pela equipe de provadores foram: aroma, consistência, sabor e impressão global.

Para realização do teste de preferência foi utilizada escala hedônica estruturada de nove pontos (CHAVES; SILVA, 2009), variando de (1) "desgostei extremamente" a (9) "gostei extremamente". Para o teste de preferência participaram 103 provadores, não treinados, de ambos 
os sexos, com idade entre 18 a 55 anos objetivando a determinação da preferência entre as formulações do "licor típico amazônico a base de açaí"analisadas.

Os provadores antes do início deste teste preencheram um questionário com perguntas sobre o consumo alcóolico e doenças preexistentes e depois foram instruídos quanto ao correto preenchimento da ficha do teste de preferência bem como o consumo da água para a limpeza do palato, o aroma do pó de café para a neutralização dos odores entre uma amostra e outra e da possibilidade de fazer sugestões no rodapé da ficha se achasse necessário.

A amostra preferida pelos consumidores, foi submetida ao teste de aceitabilidade e assim como nos estudos de Chaves e Silva (2009) foi utilizada uma ficha com escala hedônica estruturada de sete pontos, com variação de (1) "desgostou muito" a (7) "gostou muito", em relação à amostra do licor preferida. Este teste também foi realizado com 103 provadores, não treinados e de ambos os sexos, objetivando a determinação da aceitabilidade e a intenção de compra do "licor típico amazônico a base de açaí”.

\section{Caracterização Funcional}

Um dos métodos para a quantificação de antocianinas é a determinação da quantidade absoluta total deste pigmento presente num extrato particular, estimando, dessa maneira, o teor de antocianinas expresso usualmente em miligramas de antocianinas por 100 gramas de amostra como elucidado por Teixeira et al.; (2008) em seu estudo.

A metodologia utilizada para quantificação de antocianinas foi baseada em Askar \& Treptow (1993) e por ROGEZ (2000), por espectrofotometria UV/VIS diferencial em análise laboratorial e os resultados obtidos foram expressos em $\mathrm{mg}$ de antocianinas totais/L com laudo emitido pelo técnico responsável. A análise foi realizada através da fórmula:

$$
\mathrm{C}_{1}=\left(\left(\text { A } 514-\mathrm{A}_{700}\right) \mathrm{pH} 1,0-\left(\mathrm{A} 514-\mathrm{A}_{700}\right) \mathrm{pH} 4,5\right) \text { x D } \times \text { F }
$$

Onde:

$\mathbf{C} \mathbf{1}=$ Concentração em antocianinas em $\mathrm{mg} / \mathrm{Kg}$ ou de extrato;

A = Absorbância;

D = Fator de diluição do suco; e

$\mathbf{F}=18,6786$ = Fator de conversão, ligado aos coeficientes de extinção molar e peso aos coeficientes de extinção molar e peso molecular, das antocianinas do açaí (cianidina-3-rutinosídeo e cianidina-3glicosídeo). 


\section{Resultados e Discussão}

\section{Caracterização Sensorial}

(a) Teste de Caracterização

Com base nos resultados obtidos no teste sensorial de caracterização, observou-se que quanto ao atributo aroma as formulações que apresentaram maiores concentrações de álcool, F1 a F4, segundo os provadores, conferiram um aroma desagradável. Logo, as que obtiveram melhores resultados foram as formulações com maior concentração de açaí.

Quanto à consistência, as formulações com maior concentração de açaí obtiveram melhores resultados, sendo assim as formulações entre a F6 e a F8, merecem destaque. Segundo o estudo Geocze (2007) o aproveitamento de açúcares ricos em sacarose resultam em licores com maior viscosidade do que os que são feitos com açúcares ricos em glicose. As formulações com maior proporção de açaí e menor de aguardente resultaram em um volume final menor de licor do que as formulações com menor concentração de açaí. Como a quantidade de açúcar foi constante nas formulações do licor, observou-se um aumento na concentração de sacarose devido ao menor volume gerado.

Quanto ao sabor, as formulações que apresentaram um sabor mais acentuado da fruta regional, obtiveram os melhores resultados. A partir da formulação F5, o sabor do álcool não prevaleceu ao da fruta.

Quanto à impressão global, as formulações F5, F6 e F7 foram classificadas pelos provadores como sendo as melhores. Partindo dos resultados obtidos, foram em seguida submetidas ao teste sensorial de preferência e a formulação F7 obteve melhor desempenho, sendo posteriormente submetida à teste de aceitabilidade.

\section{(b) Teste de Preferência}

Os resultados obtidos neste teste, demonstraram que a amostra F7 obteve a maior média de pontos em relação às formulações F5 e F6 em todos os atributos como mostra a Tabela 2 e a Figura 2. O estudo da análise estatística demonstrou que houve diferença significativa $(p<0,05)$ entre as formulações quanto aos atributos: consistência e impressão global ( $p=0,0292$ e $p=0,0076$ ), sendo que a formulação F7 obteve as maiores médias $(7,36$ e 7,24), respectivamente. Na avaliação dos atributos: aroma e sabor, não houve diferença significativa estatisticamente, entretanto a formulação 
F7 também predominou em relação à média das notas, obtendo a maior pontuação nesses atributos $(6,64$ e 7,19$)$.

Tabela 2- Média e desvio padrão quanto aos atributos analisados durante teste de preferência do licor típico amazônico a base de açaí.

\begin{tabular}{|c|c|c|c|c|c|c|c|}
\hline & \multicolumn{2}{|c|}{ Amostra F5 } & \multicolumn{2}{|c|}{ Amostra F6 } & \multicolumn{2}{|c|}{ Amostra F7 } & \multirow[b]{2}{*}{$p$-valor $* *$} \\
\hline & Média & Dp & Média & Dp & Média & Dp* & \\
\hline Aroma & 6,24 & $\pm 1,84$ & 6,58 & $\pm 1,79$ & 6,58 & $\pm 1,84$ & 0,2302 \\
\hline Consistência & 6,72 & $\pm 1,89$ & 7,13 & $\pm 1,67$ & 7,36 & $\pm 1,67$ & 0,0292 \\
\hline Sabor & 6,63 & $\pm 1,97$ & 7,11 & $\pm 2,03$ & 7,19 & $\pm 1,88$ & 0,0902 \\
\hline Impressão Global & 6,46 & $\pm 1,92$ & 7,06 & $\pm 1,85$ & 7,24 & $\pm 1,81$ & 0,0076 \\
\hline
\end{tabular}

No estudo de Coelho et al. (2007), foram elaborados quatro formulações de licor de umbu (fruta típica do nordeste brasileiro) por maceração com quatro tipos de alcoóis sendo eles: cereal (arroz), tubérculo (batata), gramínea (cana-de-açúcar) e de gramínea desodorizada, propondo testar a preferência quanto ao flavor e aroma. A preferência foi determinada com a participação de 53 provadores utilizando-se uma escala hedônica de cinco pontos. Com relação ao atributo flavor, não houve diferença significativa estatisticamente entre as quatro formulações, porém quanto ao atributo aroma, houve diferença, e o licor macerado com cana de açúcar obteve a maior média com nota igual a "não gostei nem desgostei". Ao comparar com as formulações do licor de açaí quanto a este atributo, o estudo citado apresentou média inferior ao encontrado, com notas equivalentes a "gostei ligeiramente".

Já no presente estudo, às médias encontradas para as formulações F5, F6 e F7 ficaram entre 6,24 a 7,24, classificando como "gostei ligeiramente" e "gostei regularmente".

Geocze (2001) ao submeter amostras de licor de jabuticaba obteve resultados acima de 7,0 classificando-se como "gostei regularmente" nos cinco atributos avaliados (cor, sabor, aroma, consistência e impressão global) e não foram detectadas diferenças significativas estatisticamente entre os atributos.

Na pesquisa de Teixeira et al. (2005), os quais realizaram testes sensoriais com amostras de licor a base de banana submetidas a seis tipos de tratamento com diferentes proporções de banana, solução extratora e tempo de extração, obtiveram como resultado para os atributos sabor alcoólico e impressão global com notas $(6,7$ e 7,2) equivalentes a "gostei ligeiramente" e "gostei regularmente", respectivamente.

Já no presente estudo com o licor típico amazônico a base de açaí, o atributo sabor alcoólico não foi avaliado, porém ao comparar a nota da impressão global da formulação F6 e F7 obtiveram resultados semelhantes ao estudo de Teixeira et al.; (2005), equivalendo a "gostei regularmente". 
Figura 2: Média, desvio padrão e valor mínimo e máximo das notas referentes aos atributos avaliados isoladamente no teste de preferência do licor típico amazônico a base de açaí.
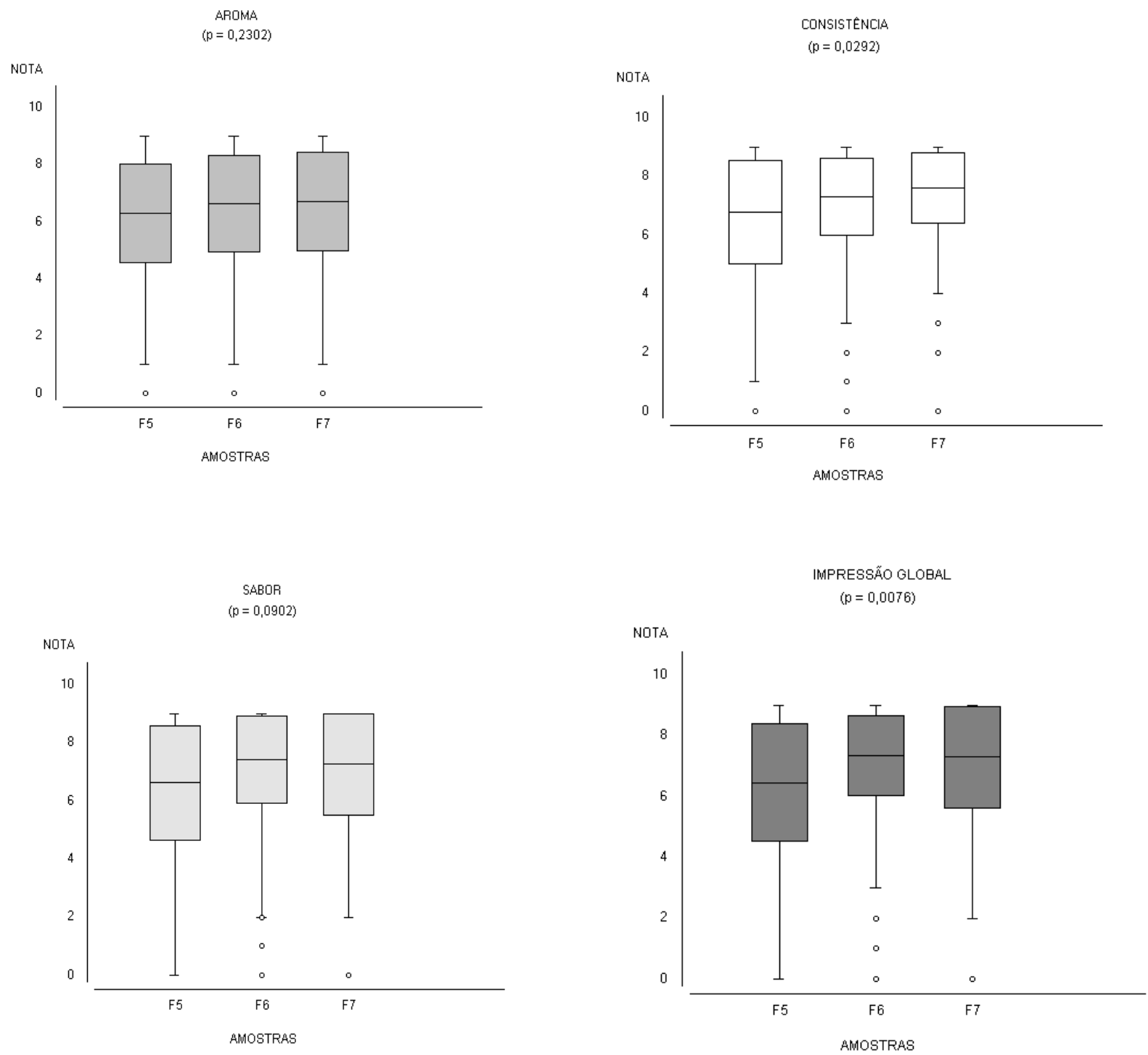

(c) Teste de Aceitabilidade

Observa-se na Figura 3, que aproximadamente 98\% ( $\mathrm{n}=101)$ dos provadores avaliaram e classificaram a amostra F7 do "licor típico amazônico a base de açaí", como "gostei ligeiramente" e "gostei muito", seguidos de 1,0\% nas notas equivalentes à "não desgostei nem gostei" e "desgostei regularmente". 
Figura 3: Distribuição da porcentagem de aceitabilidade da formulação F7do licor típico amazônico a base de açaí segundo escala hedônica de 7 pontos.

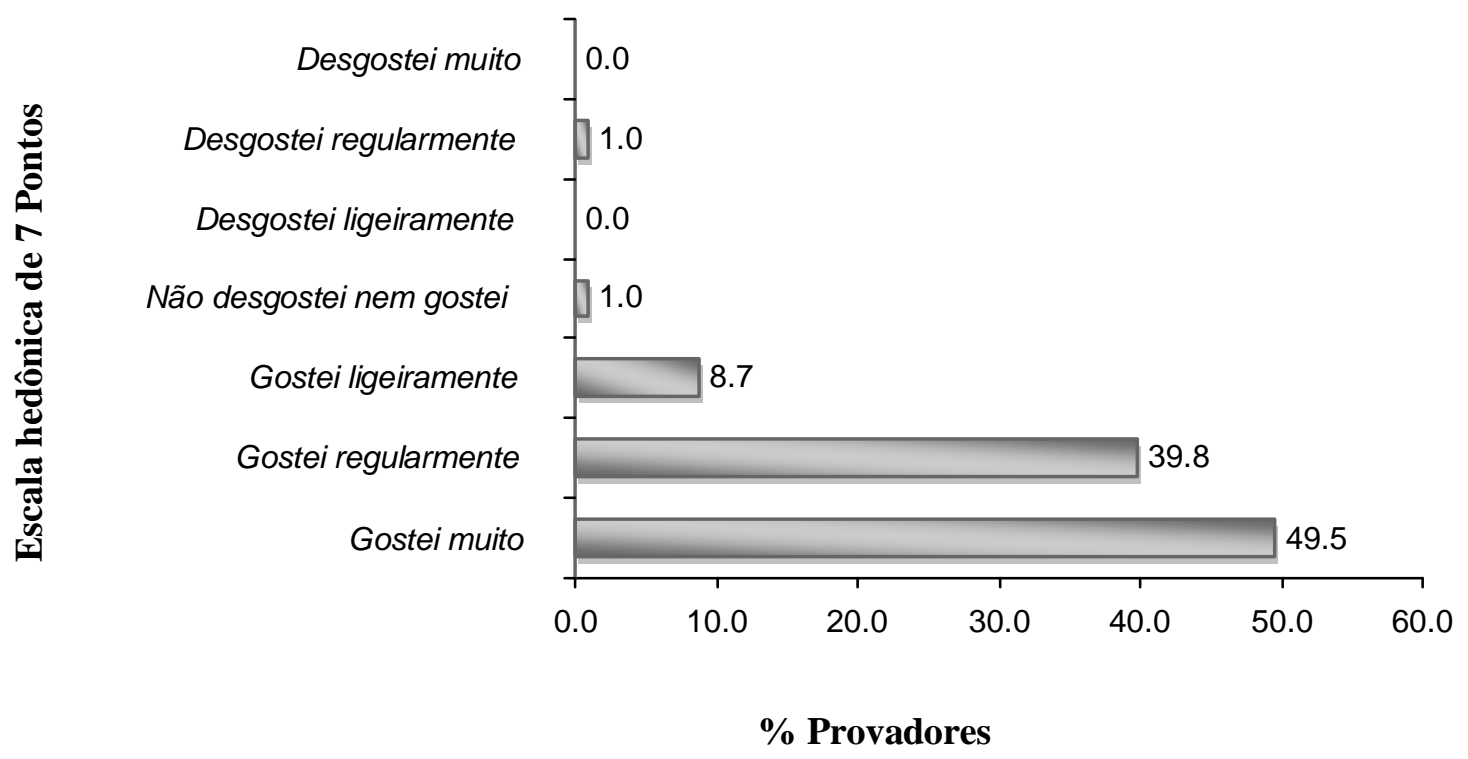

No estudo de Muniz et al. (2002), a aceitabilidade global de bebidas fermentadas a partir de frutos tropicais (ata, mangaba e ciriguela), utilizando escala hedônica estruturada de 9 pontos aplicada com 30 provadores não treinados, obtiveram notas equivalentes a "não gostei nem desgostei" para os fermentados de ata e mangaba e "desgostei ligeiramente" para o fermentado de ciriguela, notas essas menores do que a encontrada para amostra F7 do licor típico amazônico a base de açaí, que foi de "gostei muito".

Já Moura e Barrozo (1997), em sua pesquisa avaliaram sensorialmente amostras de licores a base de acerola e aguardente e de acerola e vodca, utilizando como critério de avaliação a escala hedônica de 7,0 pontos, obtiveram como resultado final 77,1\% e 78,6\% "gostei regularmente", concomitantemente. Considerados, portanto também licores com boa aceitabilidade.

No estudo de Asquieri et al. (2008) foi desenvolvido uma bebida fermentada a base de jaca a qual foi testada sensorialmente, aplicando-se escala hedônica de 9 pontos com 73 provadores. O produto elaborado apresentou um valor médio equivalente a "gostei ligeiramente" nota esta abaixo da formulação F7 do licor típico amazônico a base de açaí.

Em relação à pergunta "o que você mais gostou” do licor típico amazônico, observou-se que dentre as respostas obtidas pelos 103 provadores, aproximadamente $42 \%$ destacou o sabor, seguido de 18,5\% da suavidade do licor em relação à quantidade de álcool e 7,8\% do aroma. Já para a pergunta "o que você mais desgostou", 50,5\% dos provadores responderam "nada", seguido do teor de açúcar $29,1 \%$ e aroma $6,8 \%$. 
Quanto à intenção de compra (Figura 4), do total das 103 respostas obtidas para a pergunta "você compraria este produto", 78,6\% responderam que "sim", 19,4\% responderam "talvez" e apenas $1,9 \%$ responderam que não, comprovando a viabilidade de aquisição e consumo do produto, em escala comercial.

No estudo de Carvalho et al. (2010) que teve como objetivo elaborar um fermentado frisante de maçã com características de qualidades baseadas na sidra francesa, a aceitabilidade foi avaliada por 168 provadores com escala hedônica de 7 pontos. Observou-se nos resultados uma forte tendência de intenção de compra por parte de aproximadamente $85 \%$ dos julgadores. Apenas 6\% ficaram indecisos e menos de 8,5\% não comprariam o produto. Desta forma, a intenção de compra foi maior do que a encontrada no presente estudo, porém o percentual de negação foi maior frente ao respectivo licor.

Figura 4: Distribuição da porcentagem dos provadores em relação à intenção de compra do licor do licor típico amazônico a base de açaí.

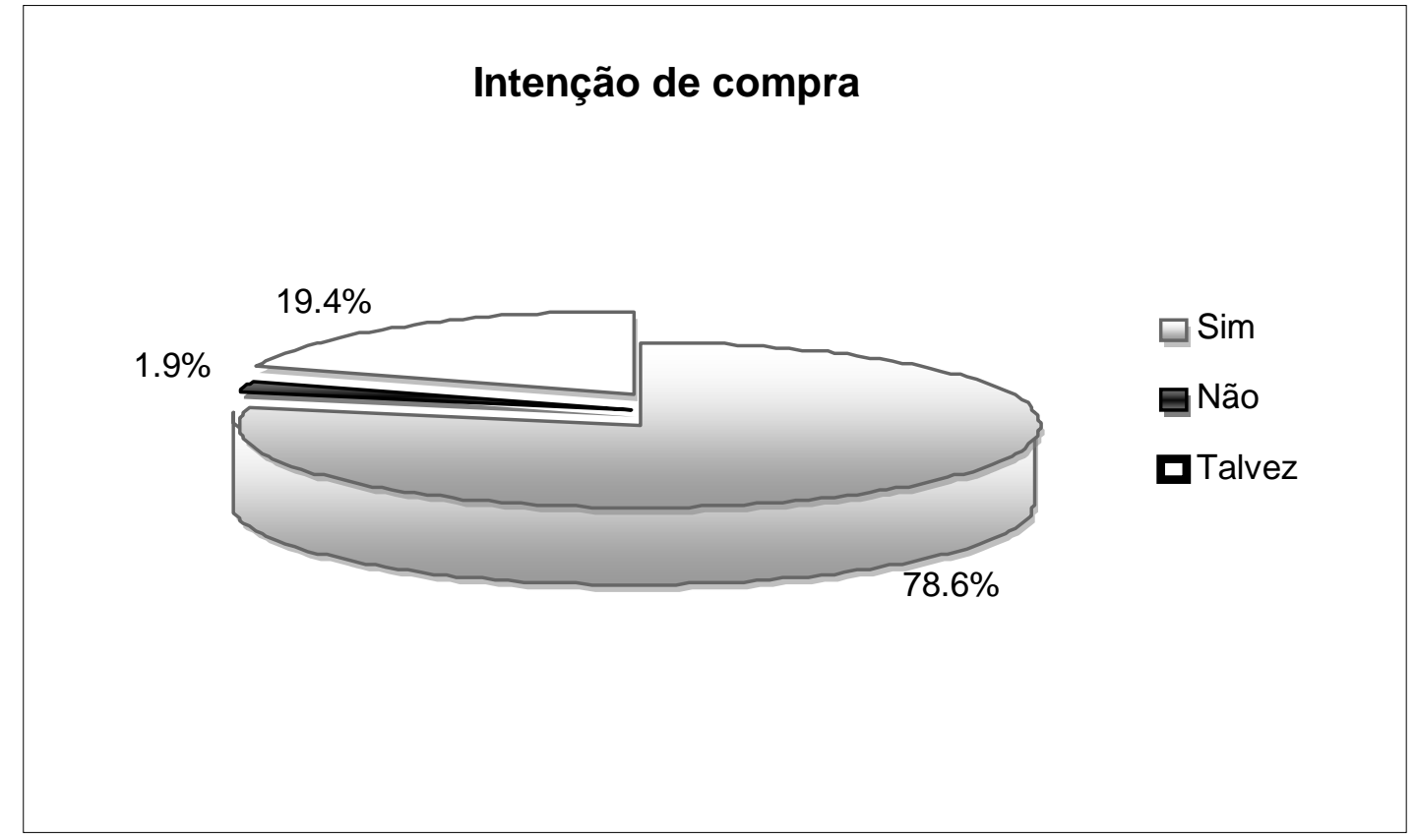

\section{Caracterização Funcional}

Foram analisadas duas formulações do licor funcional amazônico. A formulação F6 apresentou $198 \mathrm{mg} / \mathrm{L}$ de antocianina com coeficiente de variação de $1,30 \%$ e a formulação F7 o teor de antocianinas foi de $203 \mathrm{mg} / \mathrm{L}$ com coeficiente de variação em $2,11 \%$.

Em um estudo de Malacrida e Motta (2005) em que avaliaram sucos de uva tintos reconstituídos de 12 marcas de diferentes sucos de uva tinto simples (nenhuma das marcas analisadas continha informações no rótulo sobre a variedade, safra ou região produtora da uva), apresentaram uma concentração média de antocianinas $(28,70 \mathrm{mg} / \mathrm{L})$ superior à dos sucos de uva 
reconstituídos (17,31 g/L). Já no estudo de BURNS et al (2000) as concentrações encontradas em vinhos tipo Cabernet Sauvignon variaram de 11 a 32,57 mg/L, e em vinhos Merlot, variaram de 19,11 a 23,44 mg/L. Já na pesquisa de Tecchio et al. (2007), com vinho da uva tipo Bordô, encontraram teores mais elevados de antocianinas $(778,8 \mathrm{mg} / \mathrm{L})$, do que os evidenciados em outros vinhos tintos fabricados. Estes valores são atribuídos pela maior variedade de grupos de antocianinas presentes na película desta uva, aumentado assim a concentração deste pigmento.

\title{
4. Conclusão
}

Com base nos resultados obtidos nos testes sensoriais de caracterização, preferência e aceitabilidade evidenciou-se a viabilidade da produção do licor típico amazônico a base de açaí em escala comercial. E em relação à caracterização funcional, o licor apresentou melhores valores de antocianinas quando comparado com outros produtos, resultando num produto com boa fonte de compostos fenólicos.

Por fim, recomenda-se a realização de novos estudos para comparação do teor de antocianinas em bebidas e de correlação do consumo desses produtos ricos em compostos fenólicos com doenças, tais como câncer e doenças coronarianas isquêmicas.

\begin{abstract}
The acai fruit is considered an important nutritional composition with notable for its high amount of anthocyanin, a flavonoid that has beneficial effects in relation to human health, since it has antioxidant properties proven scientifically. The objective was to develop and characterize functional and sensory-based liquor with different concentration of acai acai and brandy. The samples were subjected to sensory tests of characterization, and acceptability and preference data were analyzed by ANOVA and Tukey tests at 5\% BIOESTAT using software version 5.0 (Ayres, 2007). To analyze the functional characterization, we analyzed the content of anthocyanins in accredited laboratory. Regarding the results, the testing of selected samples were characterized: F5 (50:50\%), F6 (60-40\%) and F7 (70:30\%) (acai: brandy). Then in the preference test, the samples F6 and F7 only differ in the attributes and overall consistency where the sample F7 obtained the highest average (7.12 and 7.24). This then was selected and subjected to acceptance test which got $98 \%$ acceptance among judges and $78.6 \%$ said they would buy the product. In the analysis of functional characterization, liquor F7 and F6 had anthocyanin content higher than the average found in grape juice and red wines. Thus, the results of sensory analysis showed that the product produced has a big potential consumer market and can also be classified as a beverage with good source of phenolic compounds.
\end{abstract}

Key-words: Anthocyanins; Sensory evaluation; Antioxidants.

\section{Referências}

ASKAR, A.; TREPTOW, H. Quality assurance in tropical fruit processing. New York: Springer-Verlag, 1993. 
ASQUIERI, E. R; RABÊLO, A. M. S; SILVA, A. G. M. Fermentado de Jaca: estudo das características físico-químicas e sensoriais. Revista Ciência e Tecnologia de Alimentos, v. 28, n.4, 2008.http://dx.doi.org/10.1590/S010120612008000400018

AYRES, M.; AYRES JUNIOR, M.; AYRES, D. L.; SANTOS, A. S. 2007. BioEstat 5.0 Aplicações estatísticas nas áreas da ciências biológicas e médicas. Belém, Sociedade Civil Mamirauá/CNPq.

BASHO, S.M; BIN, M. C. Propriedades dos alimentos funcionais e seu papel na prevenção e controle da hipertensão e diabetes. Revista Interbio, n. 4, 2010.

BRASIL. Decreto $\mathbf{n}^{\circ} \mathbf{2 . 3 1 4}$, de 4 de setembro de 1997. Regulamentam a lei $n^{\circ} 8.918$ de 14 de julho de 1994 , que dispõe sobre a padronização, a classificação, o registro, a inspeção, a produção e a fiscalização de bebidas. Diário Oficial da República Federativa do Brasil. Brasília, 5 de setembro 1997. Seleção1. p.19549-19555.

BURNS, J.; GARDNER, P. T.; O’NEIL, J.; CRAWFORD, S.; MORECROFT, I.; MC PHAIL, D. B.; LISTER, C.; MATHEWS, D.; MC LEAN, M. R.; LEAN, M. E. J.; DUTHIE, G. G.; CROZIER, A. Relationship among antioxidant activity, vasodilatation capacity, and phenolics content of red wines. Journal of Agricultural and Food Chemistry, v. 48, p. 220-230, 2000. http://dx.doi.org/10.1021/jf9909757

CARVALHO, J. R. F; SILVA, K. M; SIMÕES, D. R. S; WOSIACKI, G; NOGUEIRA, A. Elaboração de fermentado frisante de maçã com características semelhantes à sidra francesa. Revista Boletim do CEPPA, v. 28, n. 01, p. 97-144, 2010 .

CHAVES, C. F; SILVA, L. M. F. Análise sensorial e nutricional de preparação rica em ferro, de baixo custo, aplicada aos discentes, docentes e funcionários da Faculdade de Nutrição da Universidade Federal do Pará. Trabalho de Conclusão de Curso de Nutrição da Universidade Federal do Pará, Belém, PA, 2009.

COELhO, M. I. S; AlBUQUERQUE, L. K. S; MASCARENHAS, R. J; COELHO, M. C. S. C; SILVA FILHO, E. D. Elaboração de licores de umbu com diferentes alcoóis. II Congresso de pesquisa e inovação da rede norte nordeste de educação tecnológica, João Pessoa, PB, 2007.

GEOCZE, A.C. Influência da Preparação do Licor de Jabuticaba (Myrciaria jaboticaba Vell Berg) no Teor de Compostos Fenólicos. Trabalho de Conclusão de Curso (Mestrado em ciências de alimentos), Belo Horizonte, MG, 2007.

GRIS, E. F; FALCÃO, L. D; FERREIRA, E. A; LUIZ, M. T. B. Avaliação do tempo de meia-vida de antocianinas de Uvas cabernet sauvignon em "sorbet". Revista Boletim do CEPPA, v.22, n. 02, p. 375-386, 2004.

INSTITUTO DO DESENVOLVIMENTO ECONÔMICO SOCIAL DO PARÁ. Coordenação de Estatística Estadual (IDESP/CEE). Fórmula para cálculo de tamanho amostral. Belém, 1993.

MAFRA, T. C. S. Aspectos socioeconômicos e nutricionais do açaí. Trabalho de conclusão de curso da Universidade Federal do Pará, Belém-PA, Brasil, 2008.

MALACRIDA, C. R; MOTTA, S. Compostos fenólicos totais e antocianinas em suco de uva. Revista Ciências e Tecnologia de Alimentos, v. 25, n. 4, p. 659-664. 2005. http://dx.doi.org/10.1590/S0101-20612005000400006

MENEZES, E. M. S; TORRES A. T.; SRUR A. U. S, Valor Nutricional da polpa do açaí liofilizada, Manaus 2008.

MOURA, A. S; BARROZO, P. P. M. Licor de acerola (Malpighia punicifolia L.). Trabalho de conclusão de curso de engenharia química, Universidade Federal do Pará, Belém, 1997.

MUNIZ, C. R; BORGES, M. F; ABREU, F. A. P; NASSU, R. T; FREITAS, C. A. S. Bebidas fermentadas a partir de frutos tropicais. Revista Boletim do CEPPA, v. 20, n. 2, p. 309-322, 2002.

NETZLAFF, M. L. W; ROMAN, J.A; BRAGUETO, G. Sorvete de chocolate à base de soja: elaboração e análise sensorial. Revista Nutrição Brasil, v. 9, n. 2, p. 106-111, 2010.

ROGEZ, H. Açaí: Preparo, Composição e Melhoramento da Conservação, EDUFPA, Belém-PA, Brasil, 2000. 
SAMPAIO, P. B. Avaliação das propriedades funcionais do açaí (Euterpe oleracea) em plasma humano. Dissertação de mestrado ao programa de pós-graduação em ciências e tecnologia de alimentos, Universidade Federal do Pará, Belém, 2006.

SANTOS, G. M; MAIA, G. A; SOUSA, P. H. M; COSTA, J. M. C; FIGUEIREIDO, R. W.; PRADO, G. M. Correlação entre atividade antioxidante e compostos bioativos de polpas comerciais de açaí (Euterpe oleracea Mart). Archivos latino americanos de nutricion, v. 58, n. 2, 2008.

TECCHIO, F. M; MIELE, A; RIZZON, L. A. Composição físico-química do vinho Bordô de Flores da Cunha, RS, elaborado com uvas maturadas em condições de baixa precipitação. Ciência Rural, v. 37, n. 5, p. 1480-1483, 2007. http://dx.doi.org/10.1590/S0103-84782007000500044

TEIXEIRA, L. J. Q.; RAMOS, A. M.; CHAVES, J. B. P. Avaliação Tecnológica da Extração Alcoólica no Processamento de Licor de Banana. Revista Boletim do CEPPA, v. 23, n. 2, p. 329-346, 2005.

TEIXEIRA, L. N; STRINGHETA, P. C; OLIVEIRA, F. A. Comparação de métodos para a quantificação de antocianinas. Revista Ceres, v. 55, n. 4, p. 297-304, 2008.

Submetido em 08 dez. 2010; Revisão submetida pelos autores em 15 nov. 2011; Aceito para publicação em 29 dez. 2011. 